\title{
Mach Principle and Post-Einsteinian Relativity Theory
}

\author{
B. S. Sadykov \\ Scientific Center, Moscow State University of Printing Arts, Moscow, Russia \\ Email: sal_fir@hotmail.com
}

How to cite this paper: Sadykov, B.S. (2018) Mach Principle and Post-Einsteinian Relativity Theory. Journal of Modern Physics, 9, 35-50.

https://doi.org/10.4236/jmp.2018.91003

Received: October 20, 2017

Accepted: January 12, 2018

Published: January 15, 2018

Copyright $\odot 2018$ by author and Scientific Research Publishing Inc. This work is licensed under the Creative Commons Attribution International License (CC BY 4.0).

http://creativecommons.org/licenses/by/4.0/

\begin{abstract}
Inertia is one of the most mysterious forces of nature. Its physical nature is unknown. Accordingly, attempts are made to avoid this "dark force" in one way or another. In Newton's mechanics and in the special relativity theory, this is done by postulating inertial reference frame, in the general relativity theory by postulating the equivalence principle. Postulates cannot change the laws of nature. One needs to know the latter. Given that the forces of inertia result in non-inertial reference frames only, a hypothesis of the induction nature of inertia has been made. According to this hypothesis, the forces of inertia result as the reaction of a body to external influence. A system of equations explaining the physical nature of inertia and its connection to gravity has been drafted. A group of coordinate conversion has been suggested, which without violating the general covariance of the laws of nature, enables to distinguish one reference frame associated with one body from another system associated with another body, to determine the privileged ones, and to take into consideration their influence on the readings of measurement instruments. A modified mechanics had been created. It takes into consideration the Mach's principle and enables to cover all systems with the relativity principle. All of the relativistic effects arise from it. The reasons for emergence of new particles, dark matter, dark energy etc. are explained. New effects are predicted.
\end{abstract}

\section{Keywords}

Inertia, Gravity, Massive Reference Systems, Relativity, Inertial Field, Kinetic Field Moment, Mach's Principle, Dark Matter

\section{Introduction}

Inertia is one of the most mysterious forces of nature. It is unknown how it 
forms, where its sources are, why it is and what it is. Numerous generations of genius scientists, from Newton to Einstein and after them, have worked to uncover the mysteries of inertia but the key issue of why the forces of inertia result in non-equable and curvilinear motion and disappear in equable and rectilinear motion still remains unresolved [1].

The first scientist who attempted to comprehend the physical nature of inertia was: I. Newton. He was confident that the reason for bodies inertia was the surrounding bodies, however, conducting various experiments, he did not detect any effect and concluded that the reason for bodies inertia was caused the space itself.

Newton's concept was criticized by his contemporaries. He was criticized by physicists (Huygens), mathematicians (Leibniz), philosophers (Berkeley) etc. who believed that a limited number of surrounding bodies had been involved in Newton's experiments, and it had been required to introduce remote bodies-the stars-as the complete mass of the Universe is in them [2].

Newton knew about the stars much more than his opponents but he thought the stars were too far away to have instantaneous effect on the terrestrial processes. That is why he did not search for the reason for inertia and postulated the law of inertia. At the late 19th century, the issue of inertia was not raised by Ernst Mach. He strongly criticized Newton's mechanics, and wrote his Mechanics in which he claimed that the inertial properties of a body were determined by all the other bodies of the Universe. He did not explain how it was implemented in practice.

Mach's ideas had a profound effect on Einstein and played a major role in the GRT creation. Einstein was grateful to Mach for "inspiring ideas", named them "Mach principle" (MP) and attempted to prove them from the point of view of physics. However, he failed to accomplish it, and the general relativity theory (GRT) appeared without the MP [3].

The physical nature of inertia is unknown but attempts were made to experimentally test this principle. An overview of these works can be found in article [4]. In non-inertial reference frames (NIRFs), forces of inertia which violate the laws of energy, kinetic moment, angular momentum preservation etc. arise, and the main axiom of physics is violated: motion relativity. Accordingly, attempts are made to avoid this "dark force" in one way or another. In the special relativity theory (SRT), it is done by postulating inertial reference frames (IRFs), in the general relativity theory (GRT), by postulating the equivalence principle reducing inertia to gravity.

Postulates cannot change the laws of nature. The forces of inertia have resulted and will result, that is why one needs to know, not to mask them. Given that the forces of inertia result in the NIRFs only, we have made a hypothesis of the induction nature of inertia [5]. The physical considerations underlying this hypothesis were partly outlined earlier and will be repeated in this article shortly. Similar ideas emerged in [6] as well. 


\section{Inertia as Reaction of Space to Effect of Matter}

According to this hypothesis, any particle creating a static (potential) field in the resting state $\boldsymbol{S}_{n}$, creates a dynamic (vortex) field when in motion $\boldsymbol{D}_{n}$ [7]. The totality of these fields forms a single field of matter named the inertial field (IF). In relation to MP, this means that the celestial bodies moving relative to each other generate an IF filling in the entire space, creating a certain quasi-elastic environment which prevents from accelerated motion. The reaction of this environment to the forces seeking to change its state is the response of the Universe perceived as the inertia of the body. The IF is a special field, unlike the electromagnetic, gravitational, nuclear, and other fields, it is scalar, and it defines the fabric of the space. The property of the space is determined by some field pulse $\Pi$, so that its change induces the forces of inertia $\boldsymbol{F}_{i n}=-\mathrm{d} \Pi / \mathrm{d} t$. In the state of rest and equable rectilinear motion, the IF cannot be observed, it is the zero reference point for all measurement instruments, instruments react to its change only when the force of inertia results. The interaction between particles and the IF as defined by the field pulse is complex. We are not aware of how the global field of space is formed, it is absolute to us.

Given that the real world is four-dimensional, let us assume the IF very tentatively as a sum of products of various charges $q_{n}$ (the electric, high-rate, low-rate, gravitational etc.) of moving particles and their 4-vector potentials $A_{\mu}^{n}$

$$
\Pi_{\mu}=m_{i n} u_{\mu}=\frac{1}{c} q_{n} A_{\mu}^{n}, \Pi_{4}=\frac{i}{c} U, \mu=1,2,3,4, n=1,2, \cdots
$$

where $m_{i n}$ is the inert mass, $\boldsymbol{u}$-relative velocity, $U$ is the potential energy, summation is carried out for all types of charges by introducing the IF, the necessity to postulate the IRFs is dropped, the force of inertia is transformed into the standard induction force, and the mechanical kinetic moment $\boldsymbol{p}=m_{0} \boldsymbol{u}$ acquires the field pulse component $\Pi$ defining interaction of the body with all the other bodies, together they form the complete 4-impuls of the body

$$
K_{\mu}=p_{\mu}+\Pi_{\mu}, K_{4}=i m c=i m_{0} c(1+\varepsilon), \varepsilon=U / m_{0} c^{2}
$$

The $\varepsilon$ value which defines the fraction of potential energy within the resting energy is a form factor determining the structure (curvature) of the space of a given point. If the external forces do not affect the body, its kinetic moment remains, and the body will continue its motion at a constant 4 -velocity in the direction in which it was moving

$$
V_{\mu}=\left(p_{\mu}+\Pi_{\mu}\right) / m=\text { const, } V_{4}=i c(1+\varepsilon) / m_{0} c^{2}=\text { const }
$$

If you associate a reference frame with such a body-we named such systems massive reference frames (MRFs), it will be inertial but the relativity principle cannot apply to it while it is unknown in relation to which body it is moving and how to reconcile the data from various MRFs if they are related to different bodies. 


\section{Mach-Poincaré Relativity Principle}

Let us assume that two massive bodies, e.g. the Earth and the Sun, are given to which the MRFs $S$ and $S^{\prime}$ are related Assume that $S$ rests, and $S^{\prime}$ moves relative to it with some acceleration. In the accelerated system, the force of inertia results and does not result in the resting system. Why, if the systems are moving relative to each other?

The answer is provided by the modified MP, it stipulates that the MRFs $S$ and $S^{\prime}$ were not isolated bodies, they were among other bodies, and the body $S^{\prime}$ moved not relative to the body $S$ but relative to all the bodies of the Universe, all of them together created a single global reference system $S_{g}$, and the system $S^{\prime}$ moved relative to that global system (Figure 1).

Therefore, the forces of inertia would result in the resting system $S$ as well if $S_{g}$, i.e. the entire Universe, accelerated relative to it!

How is it possible to determine the velocity relative to $S_{g}$ if the Universe has no center or isolate point which could be accepted as the starting point? The velocity is always relative to the IF of space. In case of equable rectilinear motion, the IF is not observable, therefore an indicator-another body-is required to determine its location. If there is no other body, it is not possible to determine anyhow whether the system is resting or in the state of equable rectilinear motion (Galileo relativity principle). In case of accelerated motion, another body is not necessary, the force of inertia results, and it is possible to determine the starting point and the acceleration start.

Thus, any point of space can be assumed to be the starting point while the effect of the surrounding bodies is taken into account by the kinetic field moment, and it is measurable. The velocity relative to the IF is determined with Formula (3).

With regard to the identification of measurement instruments, it can be implemented if the scales of the instruments are calibrated as functions of $V_{\mu} / V_{4}$, i.e. of all the values affecting their readings. Then the scale becomes non-rigid and automatically changes when switching from one MRF to any other, and the relations of the values being measured remain the same in all MRFs regardless of the nature of the bodies to which they are related.

We have named this understanding as "Mach-Poincaré relativity principle" (MPRP). It states [5]: If, along with changing physical processes, readings of measurement instruments are changed simultaneously and proportionately, it is not possible to detect this measurement with any experiments.

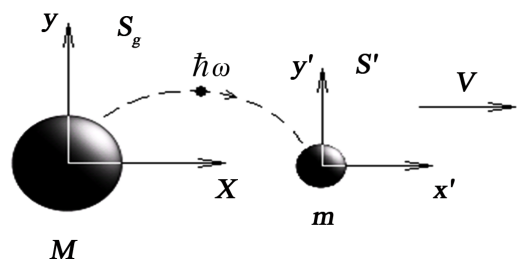

Figure 1. Connection between MRFs $S^{\prime}$ and $S_{g}$ occurs by means of light signal. 
On the basis of this principle, a generalized group of the MRF coordinate conversion was obtained, it, without violating the general covariance of the laws of nature, enables to distinguish one MRF related to one body from another system related to another body, to single out the privileged one, and to take into consideration their influence on the readings of the measurement instruments [5]

$$
\begin{gathered}
x^{\prime \mu}=\frac{x^{\mu}+i a_{v}^{\mu} x^{v}}{\sqrt{1+a_{v}^{\mu} a_{\mu}^{v}}} \exp i \gamma_{\mu}, x^{\mu}=\frac{x^{\prime \mu}-i \tilde{a}_{v}^{\mu} x^{\prime v}}{\sqrt{1+a_{v}^{\mu} a_{\mu}^{v}}} \exp \left(-i \gamma_{\mu}\right) \\
a_{\mu}^{4}=\tilde{a}_{4}^{\mu}=-a_{4}^{\mu}=c \frac{m_{0} u^{\mu}+\Pi^{\mu}}{m_{0} c^{2}+U}, \gamma_{1}+\gamma_{2}+\gamma_{3}+\gamma_{4}=0
\end{gathered}
$$

The summation convention is the standard one, summation is assumed by repeating indexes (in the one member of an equality as, e.g., index $v$ ). The coordinates of events in the MRF are unequivocally defined by the relative change in the kinetic moment energy of a signal which connects MRFs $S$ and $S^{\prime}$. If it is insignificant, then Formula (4) convert to the Galilean transformation, if it is determined by participation of the signal only in the relative motion of the "massless" MRF, to Lorentz transformation. In all other cases, the MRFs are distinguishable, and their influence on processes varies.

Ratios $a_{v}^{\mu} \sim \Pi / U$ never vanish, this means that neither perpetual rest not perpetual motion exists in the nature, everything is relative.

\section{Change of Scale of Measurement Instruments When Changing from One Massive Reference Frame to Another}

Assume that two massive bodies to which MRFs $S$ and $S^{\prime}$ (Figure 1) are related are given, and in each system a researcher is present and measures some value and informs his/her colleague from the other system about the results of his/her measurement.

Information is exchanged with a light signal. Receiving his/her colleague's signal, each of the researchers compares it to his/her measurement data and recognizes that they are identical.

Now, the colleagues have changed their places and make the same measurements but with the instruments of their own systems. They find out that the data differ by $\varepsilon=\left|\varphi_{S}-\varphi_{S^{\prime}}\right| / c^{2}$, where $\varphi$ is the gravitational potential of the MRF because they are calibrated in different systems. To determine the effect of the MRF proper, the measurement instruments should be calibrated at the same point. Using (3), let us define duration by the fourth coordinate

$$
\mathrm{d} x^{\prime 4}=V^{4} \mathrm{~d} t=i c(1+\varepsilon) \mathrm{d} t=(1+\varepsilon) \mathrm{d} x^{4}, \mathrm{~d} \tau=(1+\varepsilon) \mathrm{d} t
$$

Assume that the speed of light is constant, therefore $\mathrm{d} \tau=(1+\varepsilon) \mathrm{d} t$ to determine the interspace we will posit that the 4-volume invariant $d x^{\prime 1} d x^{\prime 2} d x^{\prime 3} d x^{\prime 4}=d x^{1} d x^{2} d x^{3} d x^{4}$. Given (6), we have

$$
\mathrm{d} x^{\prime 1} \mathrm{~d} x^{\prime 2} \mathrm{~d} x^{\prime 3}=\frac{\mathrm{d} x^{1} \mathrm{~d} x^{2} \mathrm{~d} x^{3}}{1+\varepsilon}=\frac{\mathrm{d} x^{1}}{1+\varepsilon_{1}} \frac{\mathrm{d} x^{2}}{1+\varepsilon_{2}} \frac{\mathrm{d} x^{3}}{1+\varepsilon_{3}}
$$


When $S$ changes to $S^{\prime}$, the body volume seems to deform, each side changes as $\mathrm{d} x^{p}=\left(1+\varepsilon_{p}\right) \mathrm{d} x^{\prime p}$. The possibility of space anisotropy is taken into consideration in this case. For homogeneous space, $\varepsilon_{p}=\varepsilon / 3$. It is assumed that the systems are motionless.

Consider the same effects in a moving system. Measure the linear size of the body in the direction of motion, measurement is carried out simultaneously $\Delta x^{4}=0$, of (4) we have

$$
\begin{gathered}
\Delta x^{\prime p}=\frac{\Delta x^{p} /\left(1+\varepsilon_{p}\right)}{\sqrt{1-a_{v}^{p} \tilde{a}_{v}^{p}}}=\frac{\Delta x^{p}}{\sqrt{\left(1+\varepsilon_{p}\right)^{2}-b_{v}^{p} \tilde{b}_{v}^{p}}}, p=1,2.3 \\
b_{4}^{p}=\left(1+\varepsilon_{p}\right) a_{4}^{p}=\left(u^{p}+\Pi^{p} / m_{0}\right)^{2} / c^{2},(p \neq v)
\end{gathered}
$$

The sizes of the bodies in the direction of motion seem to reduce, singularity appears in powerful fields $\varepsilon_{p}=-1$, the source of light does not reach the receiver.

Consider the time interval

$$
\Delta x^{\prime 4}=\frac{\Delta x^{4}(1+\varepsilon)+i a_{p}^{4} \Delta x^{p}}{\sqrt{1-a_{v}^{p} \tilde{a}_{v}^{p}}}
$$

During time $\Delta x^{4}$, the moving system covers distance $\Delta x^{p}=V^{p} \Delta \tau=-i a_{4}^{p}(1+\varepsilon) \Delta x^{4}$. This gives

$$
\Delta x^{\prime 4}=\Delta x^{4} \sqrt{(1+\varepsilon)^{2}-b_{p}^{4} \tilde{b}_{p}^{4}}
$$

The passage of time in the moving system seems to slow down. In this case, unlike in the case of the SRT, the change concerns not the complete system but only the light signal, therefore no anomalies like "clock paradoxes" result in this case. The signal is affected by the gravitational field of reference body $\varphi=-\gamma m / r$, and the signal, leaving the source field, reaches the receiver field and does work $A=U_{S}-U_{S^{\prime}}$, its trajectory curves

$$
r_{2}^{\prime}-r_{1}^{\prime}=\int \frac{\mathrm{d} r}{1+\varepsilon} \approx \int \mathrm{d} r\left(1+\frac{\gamma m}{r}\right)=r_{2}-r_{1}+\frac{\gamma m}{c^{2}} \ln \frac{r_{2}}{r_{1}}
$$

Time $\mathrm{d} t=\mathrm{d} r / c$ is required to cover the distance or

$$
t_{2}^{\prime}-t_{1}^{\prime}=\int\left(1-\frac{\gamma m}{c^{2} r}\right) \frac{\mathrm{d} r}{c}=t_{2}-t_{1}-\frac{\gamma m}{c^{2}} \ln \frac{t_{2}}{t_{1}}
$$

The GRT gives the same results but the effects are interpreted differently in the case.

\section{Energy and Kinetic Moment of Particle in Inertial Field}

In determining the kinetic moment, we proceeded from the quasi-classic approximation (3), which is not quite correct, as this approximation is not relativistic and permits action at a distance. The definition should be based on conversion (4) 


$$
\begin{aligned}
& K^{\prime \mu}=m^{\prime} \frac{\mathrm{d} x^{\prime \mu}}{\mathrm{d} t^{\prime}}=i m^{\prime} c^{\prime} \frac{\mathrm{d} x^{\prime \mu}}{\mathrm{d} x^{\prime 4}}=\Omega_{\mu}^{\mu}\left(K^{\mu}+i a_{v}^{\mu} K^{v}\right) \\
& \Omega_{\mu}^{\mu}=\frac{1}{\sqrt{1+a_{\mu}^{v} a_{v}^{\mu}}}=\frac{1}{\sqrt{1-a_{v}^{\mu} \tilde{a}_{v}^{\mu}}}, \mu \neq v=1,2,3,4
\end{aligned}
$$

Formula (11) defines the law of the kinetic-moment change, and the kinetic motion equals to

$$
K^{\mu}=\Omega_{\mu}^{\mu}\left(m_{0} u^{\mu}+\Pi^{\mu}\right)=\Omega_{\mu}^{\mu} m c a_{4}^{\mu}, K^{4}=\Omega_{3}^{4} m c a_{4}^{4}
$$

This results in invariant

$$
K_{\mu} K^{\mu}=\frac{m c a_{1}^{4}}{\sqrt{1-a_{v}^{1} \tilde{a}_{v}^{1}}} \frac{m c a_{4}^{1}}{\sqrt{1-a_{v}^{1} \tilde{a}_{v}^{1}}}+\cdots+\frac{m c a_{4}^{4}}{\sqrt{1-a_{4}^{p} \tilde{a}_{4}^{p}}} \frac{m c a_{4}^{4}}{\sqrt{1-a_{4}^{p} \tilde{a}_{4}^{p}}}=-m^{2} c^{2}
$$

where $E_{0}$ is the energy of a particle resting in the potential field $U$. Given the relation

$$
K_{p}=-K^{p}, \quad K_{4}=K^{4}=i E / c
$$

we determine the square of the kinetic-moment energy of the particle in the IF

$$
c^{2}(\boldsymbol{p}+\Pi)^{2}-E^{2}=-E_{0}^{2}, E_{0}=m c^{2}=m_{0} c^{2}+U
$$

$m_{0} c^{2}$ is assumed as the starting point, let us transfer it to the zero point

$$
W=E-m_{0} c^{2}=\frac{m_{0} c^{2}+U}{\sqrt{1-\boldsymbol{a} \tilde{\boldsymbol{a}}}}-m_{0} c^{2}
$$

For weak fields and low velocities $(\boldsymbol{a} \tilde{\boldsymbol{a}} \ll 1)$ and (17) converts to the classical energy

$$
W \approx\left(m_{0} c^{2}+U\right) /\left(1+\frac{1}{2} \boldsymbol{a} \tilde{\boldsymbol{a}}\right)-m_{0} c^{2}=\frac{(\boldsymbol{p}+\Pi)^{2}}{2 m}+U
$$

For an electrically charged particle $\Pi=-e \boldsymbol{A} / \boldsymbol{c}$, in this approximation (18) is widely used in the electrodynamics. These formulas differ from the respective formulas of the SRT by presence of $(\Pi, U)$ potentials and require no additional comment. We would like to draw attention to the mass dependence.

\section{Free and Bound Mass}

As seen in (17), mass is a complex function, it depends not only on the velocity but is also a function of interaction energy in general, therefore it is not excluded that, observing the same particle in different energy conditions, we assume it to be different ones.

Mass consists of two components: material (free) $m_{0}$ and field (bound) $m_{\text {in }}$ mass. Gravitational and inert masses exist but they will be discussed below. Material mass expresses the quantity of matter in the particle volume and does not depend on anything; inert mass is not constant; it is induced by the inertial field as the reaction of space. The particle at a point with potential energy $U_{n}$ has mass $m_{n}$, when converting to a point with potential energy $U_{k}$, its mass will change to 


$$
\Delta m=m_{n}-m_{k}=\left(U_{n}-U_{k}\right) / c^{2}
$$

The reason is that every environment, including the IF, has the maximum possible transmission capacity characterized by some critical velocity $c_{k}$ which it is impossible to exceed under the given conditions. If a particle has somehow received energy $E$ exceeding its limit kinetic energy $E_{k}$, then excessive energy results $\Delta E=E-E_{k}$, it cannot be used to increase the velocity, it either converts to another type of energy or a new particle forms from it.

For compound particles (a molecule, atom, nucleus etc.), one of the possible methods to discharge excessive energy is to divide into the smaller fractions. If it is divided into $n_{1}$ particles with masses $m_{1}, n_{2}$ particles with masses $m_{2}$ etc., the sum of the fraction masses does not correspond to the source mass, the $\Delta m=n_{n} m_{n}-m$ defect results which is the measure of bond energy

$$
\Delta E=-\Delta U=\left(n_{n} m_{n}-m\right) c_{k}^{2}
$$

The defect results in any reaction-physical, chemical, biological, nuclear etc., and its value depends on the bond energy. For a chemical reaction $\Delta E$ it is a few electron volts which, compared to the nuclei bond energy of hundreds of giga electron volts, is insignificant, therefore the mass defect is difficult to observe. If systems with a more rigid bond than the nuclear bond exist, then it is possible to exceed the "speed of light" $c_{k}>c$ but this does not conflict any laws because $c$ and $c_{k}$ are not anyhow related to the actual velocity, they are standard environment constants which have the velocity dimension.

\section{Particle Dynamics in Inertial Field}

The reason for accelerated motion is force, it is defined by the kinetic-moment change over time, however, time depends on the coordinates, such representation of time in the definition of force makes it non-relativistic. To eliminate this dependence, let us introduce the invariant time $\mathrm{d} \tau=\mathrm{d} s / c$, and differentiating (13), we have

$$
F_{\mu}=\frac{\mathrm{d}}{\mathrm{d} \tau} \frac{\left(m_{0} u_{\mu}+\Pi_{\mu}\right)}{\sqrt{1-a_{v}^{\mu} \tilde{a}_{v}^{\mu}}}=\frac{\mathrm{d}}{\mathrm{d} \tau} \frac{m c a_{\mu}}{\sqrt{1-a_{v}^{\mu} \tilde{a}_{v}^{\mu}}}, \mu \neq v
$$

It is the generalized Minkowski force which is the basis of the new mechanics, the mechanics of global systems (MGS). The word "global" emphasizes that this power takes into consideration MP, effect of the surrounding bodies but the bodies move in the IFs of other bodies and generate a field themselves, therefore motion for the field should be made as well. We will proceed from the principle of least action and compose a Lagrangian $L$. It consists of three parts, a Lagrangian of matter $L_{m}$, a field $L_{f}$, and their interaction $L_{m f}$ If the environment does not contain another type of matter, except the IF, then $L_{m}$ and $L_{m f}$ can be combined. Let us assume them as

$$
L_{m}+L_{m f}=-\sum c^{2} K_{\mu} K^{\mu}=\sum c^{2}(\boldsymbol{p}+\Pi)^{2}-\left(m c^{2}+U\right)^{2}
$$


Summation is done for all particles in the system. The Lagrangian of the field $L_{f}$ cannot be made of discrete values, the field, the continuum, and values should be continuous. Let us introduce the stress tensor of the IF

$$
G_{\mu v}=\partial_{\mu} \Pi_{v}-\partial_{v} \Pi_{\mu}+\frac{i}{\hbar}\left[\Pi_{\mu}, \Pi_{v}\right],\left(\partial_{\mu}=\partial / \partial x_{\mu}\right)
$$

with components

$$
G_{(\mu \nu)}=\left|\begin{array}{cccc}
0 & R_{z} & -R_{y} & -i Q_{x} / c \\
-R_{z} & 0 & R_{x} & -i Q_{y} / c \\
R_{y} & -R_{x} & 0 & -i Q_{z} / c \\
i Q_{x} / c & i Q_{y} / c & i Q_{z} & 0
\end{array}\right|
$$

Two invariants result from the components

$$
\begin{aligned}
& I_{1}=-\frac{1}{2} \int G_{\mu \nu} G^{\mu v} \mathrm{~d} V=\int\left(\boldsymbol{Q}^{2}-c^{2} \boldsymbol{R}^{2}\right) \mathrm{d} V, \\
& I_{2}=-\frac{1}{2} \int e_{\alpha \beta \mu \nu} G^{\alpha \beta} G^{\mu v} d V=\int c \boldsymbol{Q} \boldsymbol{R} d V
\end{aligned}
$$

where $e_{\alpha \beta \mu v}$ are Levi-Civita symbols, they equal zero if any two indices coincide and \pm 1 if all of them are different. Let us form the Lagrangian of field of these invariants

$$
L_{f}=b\left(I_{1}^{2}+I_{2}^{2}\right)
$$

where $b$ is a certain constant. Using Euler-Lagrange equation, we have a system of calibration Equation (6)

$$
\begin{aligned}
& F_{\mu}=G_{\mu \nu} V^{\nu}, \mu, \nu, \lambda=1,2,3,4 \\
& c^{-2} Y^{\mu}=\partial_{\nu} G^{\mu \nu}, Y^{\mu}=j^{\mu}+(\sigma / m) \Pi^{\mu} \\
& \partial_{\mu} G^{\nu \lambda}+\partial_{\nu} G^{\lambda \mu}+\partial_{\lambda} G^{\mu \nu}=0
\end{aligned}
$$

The first equation is a particle motion equation in the four-dimensional form, the second one defines the IF of matter flow $Y^{\mu}$, and the third one results from the first two. All of them are composed of the kinetic field momentum and its derivatives. The kinetic field momentum generated by the flow of matter (a generalized charge flow) $j_{\mu}(r, t)$ is determined by the equation

$$
\begin{aligned}
& \nabla^{2} \Pi^{\mu}-\frac{1}{c^{2}} \frac{\partial^{2} \Pi^{\mu}}{\partial t^{2}}+\frac{\sigma \Pi^{\mu}}{m_{0} c^{2}}=-\frac{1}{c^{2}} j^{\mu}, \\
& j^{\mu}=\sigma u^{\mu}, \sigma=\left(k_{n} q_{n}\right) \rho_{n}, n=1,2, \cdots
\end{aligned}
$$

where $\sigma$ and $\boldsymbol{j}$ are the density and flow of charges $q_{n}, k_{n}$ is the bond constant). The bond between the field components is determined by "Lorentz condition".

$$
\partial_{\mu} \Pi^{\mu}=0, \Pi^{4}=m_{i n} u^{4}=i U / c
$$

where $m_{i n}$ is the so-called "induced mass", this condition, in effect, expresses the law of the field pulse preservation. Let us rewrite it as

$$
\operatorname{div} \Pi=-\frac{1}{c^{2}} \frac{\partial U}{\partial t}=-\frac{\partial m_{i n}}{\partial t}
$$


As it can be seen, the source of the IF and the kinetic field momentum is the variable induction mass $m_{i n}$, it induced by the field, is "born" as the reaction of the IF space to the actions of matter. The dynamic field does not create constant mass. The process of reciprocal change of the kinetic field moment induces mass. Mass change creates a kinetic field moment. Constant conversion of the matter to the field and of the field to the matter occurs.

Let us go back to Equations (27). The spatial components of the stress tensor determine the force affecting the particle. The temporal ones determine its power

$$
\begin{gathered}
\boldsymbol{F}=\boldsymbol{Q}+\boldsymbol{V} \times \boldsymbol{R}, \mathrm{d} E / \mathrm{d} t=\boldsymbol{Q} \boldsymbol{V} \\
\boldsymbol{Q}=-\frac{\partial \Pi}{\partial t}-\nabla U, \quad \boldsymbol{R}=\operatorname{rot} \boldsymbol{\Pi}
\end{gathered}
$$

The body in the IF is affected by two forces, one of which is longitudinal and is composed of two heterogeneous forces-inertia and potential force $\boldsymbol{Q}$, the second force is transverse and also consists of the sum of two other forces-Coriolis and centrifugal force. These forces are known as the forces of inertia which occur in NIRFs, however, in this case they have a different meaning, they explain the reason for accelerated motion-the inertial field. The axial force imparts radial acceleration to the body, the lateral force imparts axial acceleration. Is accelerated expansion of the Universe not related to this, ignoring the IF, we are forced to postulate different phantom particles? This option is not excluded.

\section{Superposition of Partial Fields}

The MGS equations in this general four-dimensional form are not convenient for practical applications, and it is more convenient to single out the field of each source. In powerful fields, this cannot be done, in weak fields, in approximation (1), it is possible, and (27) is split into a system of partial equations.

$$
\begin{aligned}
& \boldsymbol{Q}=q_{n} \boldsymbol{S}_{n}=q_{n}\left(k_{n} \boldsymbol{G}_{n}\right), \quad \boldsymbol{R}=q_{n} \boldsymbol{R}_{n}=q_{n}\left(\chi_{n} \boldsymbol{D}_{n}\right) \\
& \operatorname{rot} \boldsymbol{S}_{n}=-\frac{\partial \boldsymbol{R}_{n}}{\partial t}, \operatorname{div} \boldsymbol{R}_{n}=0 \\
& \operatorname{rot} \boldsymbol{D}_{n}=\frac{\partial \boldsymbol{G}_{n}}{\partial t}+\boldsymbol{Y}_{n}, \quad \operatorname{div} \boldsymbol{G}_{n}=\rho_{n}
\end{aligned}
$$

The fields of all charges are described with the same equations but with different bond constants $k_{n}$ and $\chi_{n}$. The relation of these constants determines the rate of distribution of this field

$$
c_{n}=\sqrt{k_{n} / \chi_{n}}
$$

Knowing the relevant charge, $q_{n}$ the other parameters can be calculated, if the field is created by an electric charge $q_{1}=e$, then (33) converts to Maxwell equations, if by a gravitational charge $q_{2}=g$, then to a similar system of equations for gravidynamics

$$
\begin{aligned}
& \operatorname{rot} \boldsymbol{\Gamma}=-\frac{\partial \boldsymbol{\Omega}}{\partial t}, \operatorname{div} \boldsymbol{\Omega}=0, \boldsymbol{\Omega}=\varsigma \boldsymbol{I} \\
& \operatorname{rot} \boldsymbol{I}=\frac{\partial \boldsymbol{G}}{\partial t}+\boldsymbol{j}, \quad \operatorname{div} \boldsymbol{G}=-\rho, \Gamma=4 \pi \gamma \boldsymbol{G}
\end{aligned}
$$


The fate of these equations is dramatic, they have been suggested numerous times (Maxwell, Hertz, Heaviside, Brillouin etc.) but have not been acknowledged. Various reasons are named: absence of the negative gravitational charge, mass dependence on velocity, inability of the linear theory to explain the wobbling of the planet orbits etc. [8].

But the actual reason was not them, the equations are invariant relative to the charge sign, the sign is important to describe the interactions and is taken into account by the induction vector $\boldsymbol{\Omega}$. The real reason was the uncertainty of the gravimagnetic field (GMF) I, more precisely, the phenomena caused by its effect. The world was well described without it, and there was no need in it. Then why write it once again if they are not required?

By introducing the IF, the situation changed, it was established that the GMF existed and was, in fact, another name of the IF. The gravitational and inertial fields are interrelated, induce each other and together form a single gravi-inertial field (GIF) similar to the electromagnetic field, which propagates in the form of is distributed in the form of cross waves at the transverse speed

$$
c_{g}=\sqrt{4 \pi \gamma / \varsigma}
$$

where $\gamma$ and $\varsigma$ are the gravitational and inertial constants. This velocity is unknown but there is some circumstantial evidence of the fact that it coincides with the speed of light $c=3 \times 10^{8} \mathrm{~m} / \mathrm{s}$ in the vacuum. By accepting this as a model, we define the vacuum IF constant

$$
\varsigma_{0}=\frac{4 \pi \gamma}{c^{2}}=\frac{4 \times 3.14 \times 6.67 \times 10^{-11}}{9 \times 10^{16}}=9.3 \times 10^{-27} \mathrm{~m} / \mathrm{kg}
$$

This value is insignificant. Its insignificance explains why the GIF of standard bodies is not observed. It is significant on the scale of the Universe and plays an important role in shaping its structure. Some Effects of Gravidynamics:

\section{1) Orbital Oscillation of Planets}

Assume that an object has created a powerful GMF $\boldsymbol{\Omega}$ and a massive body is located in the center of its lines of field, e.g. the Sun. Let another, less massive, body pass by the massive body, moving at some speed. Two force will affect it: the gravitational field of the massive body $U=-\gamma m M / r$ and the inertial field $\boldsymbol{\Omega}$. The effect of these forces will change the body velocity

$$
V=\frac{\Omega r}{2}+\sqrt{\left(\frac{\Omega r}{2}\right)^{2}+\frac{\gamma M}{r}}
$$

The value of this velocity depends on the relation of the forces, if the gravitational field is powerful, it captures the body, and it will rotate around the massive one with the orbital velocity $u=(\gamma M / r)^{1 / 2}$, if the GMF is powerful, the body exits the field of the massive body, having deflected by a small angle from the initial direction. Assume that the gravitational field is powerful, it captures the body, and it will start to rotate around the massive body.

Let us select plane $z$ =const as the orbit plane and determine the coordinate system so that the GMF direction coincides with that axis $z \quad\left(\Omega=\Omega_{z}\right)$. To de- 
fine a trajectory, the kinetic field moment should be known, it includes the kinetic moment of the body $\Pi_{i}$ and of the external source $\Pi_{t}$

$$
\boldsymbol{\Pi}^{\prime}=\Pi_{i}+\Pi_{e}=\boldsymbol{R}^{\prime} \times \boldsymbol{r}, \boldsymbol{R}=m \boldsymbol{\Omega}
$$

Let us select the coordinate system so that axis $z$ coincides with the GMF direction, then $F_{x}=-\frac{\partial \Pi_{x}}{\partial t}-\frac{\partial U}{\partial x}+\left(u_{y}+\frac{1}{m} \Pi_{e}\right) R_{z}=2 u_{y} R_{z}+\frac{1}{m} R_{z}^{2} x-m \omega_{0}^{2} x$ etc.

Assuming $R_{z}=m \Omega_{z}$, we get

$$
\begin{aligned}
& \ddot{x}=2 \Omega_{z} \dot{y}+\Omega_{z}^{2} x-\omega_{0}^{2} x, \\
& \ddot{y}=-2 \Omega_{z} \dot{x}+\Omega_{z}^{2} y-\omega_{0}^{2} y, \\
& \ddot{z}=-\omega_{0}^{2} z+\omega^{2} z,\left(\omega_{0}^{2} r^{3}=\gamma M\right)
\end{aligned}
$$

The last equation expresses Newton's law and defines the dynamics of the celestial bodies in the stationary condition. The other two equations depend on $\boldsymbol{\Omega}$ and have a specific solution: $x=a \mathrm{e}^{i \omega t}, y=b \mathrm{e}^{i \omega t}$, substituting in (40) provides

$$
\omega_{1}=\omega_{0}+\Omega_{z}, \omega_{2}=\omega_{0}-\Omega_{z}
$$

As it appears from this solution, the body moves not along a smooth orbit but oscillates in relation to the orbit plane with amplitude $\pm \boldsymbol{\Omega}$. Such nutations are known, they can be checked on the Earth as well, e.g. by throwing various bodies from high altitude, from aircraft. In case of falling in the gravitational field of the Earth bodies gain the so-called "moment of inertia" $r \times \Pi$ and begin to rotate at angular velocity

$$
\boldsymbol{\omega}=\boldsymbol{\Omega}=\operatorname{rot} \Pi / m
$$

Upon measuring $\omega$ and the rotation radius, it is possible to assess the Earth's IF. It is known that all spacecraft eventually lose stability of their flight, run off their course, and begin to rotate. This seems to be the case with such spacecraft as the Pioneer, in the empty space the direction of flight changed, and it began to rotate under the influence of the IF. Examine some more examples.

\section{2) Dark Matter and Dark Energy}

As early as the thirtieth years of the previous century, astronomers noticed that the velocity of some stars is so significant that the mass of the clusters they rotate around is not enough to keep them. As the stars do not leave their orbits, a hypothesis of dark matter $m_{d}$ complementing the ordinary matter $m$ prior to executing Kepler law was made $\omega^{2} r^{3}=\gamma\left(m+m_{d}\right)$.

In 1998, it was discovered that the velocities of some galaxies were independent from the orbital radius, and a hypothesis was put forward concerning accelerated expansion of the Universe. To explain this, it was necessary to postulate the dark energy.

The Universe is enormous. It is possible that unknown fields and particles exist but it is highly doubtful that the nature required invisible particles only to create gravitational ballast. Can it happen that the existing theories do not reflect the reality accurately? This option is not excluded because the fundamental 
property of space is ignored-effect on the proceeding processes.

Let us calculate the GMF of a rotating body. Let a body with mass $M$ rotate in a closed orbit with angular velocity $\omega$. When rotating, it creates a GMF in the center of the orbit

$$
\boldsymbol{\Omega}=\frac{\varsigma M}{4 \pi r^{3}}(\boldsymbol{r} \times \boldsymbol{u})=\frac{\varsigma M}{4 \pi r^{3}}(\boldsymbol{r} \times \boldsymbol{\omega} \times \boldsymbol{r})=\frac{\varsigma M}{4 \pi r}(\boldsymbol{\omega}-(\boldsymbol{n} \boldsymbol{\omega}) \boldsymbol{n})
$$

If in this field another body is located, the field will carry it, and the body will move along be enthralled, and it will move around the GMF lines of field at velocity

$$
V=|\boldsymbol{\Omega} \times \boldsymbol{r}|=\frac{\varsigma M}{4 \pi} \omega \sin \theta
$$

This velocity depends on the mass and angular velocity of the massive body as the GMF source only and does not depend on the small-body position in relation to the large one. This velocity was observed by the astronomers in 1998. It should be noted that this is a long process, the GMF will carry the body until its angular velocity $\omega$ reaches $\boldsymbol{\Omega}$. In this case, $c_{g}$ coincides with the orbital velocity and (41) converts to Newton's law $4 \pi r=\varsigma M$. Knowing the orbit radius, we can calculate the mass of the central body. Assume that a body is on the edge of the Universe $\left(R \sim 10^{26} \mathrm{~m}\right)$ and rotates. For its orbit to be stable, it is necessary that the mass of the central body equals

$$
M=\frac{4 \pi R}{\xi} \approx \frac{4 \pi \times 10^{26}}{0.93 \times 10^{-26}} \approx 1.3 \times 10^{53} \mathrm{~kg}
$$

This mass is the same as the assumed mass of the Universe. If it is distributed evenly throughout the complete volume of space, its matter density will be $\rho \sim 10^{-26} \mathrm{~kg} / \mathrm{m}^{3}$, These data can be reached also on the basis of Equation (28), it contains term

$$
r_{D}^{-2}=\sigma / m_{0} c^{2}=-\varsigma \rho
$$

where $r_{D}$ is the Debye radius of shielding, presence of particles limits the interaction radius if the density of the Universe matter is assumed as $R=r_{D}$.

These anomalies create another paradox. The Universe expands, and the density of its matter does not change, it is possible in two cases, either the Universe is not alone and is located among other worlds, or the matter fields converts to weighable matter and vice versa. Constant density requires

$$
\frac{\mathrm{d} m}{\mathrm{~d} t}=\frac{\mathrm{d}}{\mathrm{d} t}\left(\frac{4 \pi}{3} \rho R^{3}\right)=4 \pi \rho R^{2} \frac{\mathrm{d} R}{\mathrm{~d} t}=4 \pi \rho R^{3} H
$$

$\mathrm{d} R / \mathrm{d} t=H R$ is the Universe expansion velocity, it is proportionate to the distance to star $R$, performing integrating

$$
M=M_{0} \mathrm{e}^{3 H t}=M_{0}(1+3 H t+\cdots)
$$

Assuming $H=71 \pm 4 \mathrm{~km} / \mathrm{s} \cdot \mathrm{Mpc}$, and initial mass $M_{0} \approx 2 \times 10^{53} \mathrm{~kg}$, we get

$$
\frac{\Delta M}{\Delta t} \approx 3 H M_{0} \approx 1.38 \times 10^{36} \text { кг/сек } \approx 7 \times 10^{5} M_{S} / c
$$


To preserve homogeneity of the space, the mass inflow should be about a million solar masses per second. At the scale of the Universe, this value is insignificant, but it should be obtained from somewhere. Two sources are possible: either the Universe is not closed and is located among other worlds, or processes which create masses happen in it. The first option is preferable, the Universe is located in the IF of other worlds, it is affected by force (31), and it completes complex movement under the influence of this force. But the second option cannot be dismissed according to (29) and (30), every change of the potential field equals the birth of a mass, the field converts to the matter constantly and vice versa. In nature, everything moves and changes, particles and fields are born and vanish, and sounds are produced. We cannot hear the songs of the stars and the sound of their orchestra but they exist and play an important role and should be included in the description of the dynamics of the celestial bodies. For this equation, motion should be quantized.

\section{Quantized Inertial Field}

The system of the MGS is linear and its quantizing is simple, replacing the kinetic moment with operator $p_{\mu} \rightarrow-i \hbar \partial_{\mu}$ in (22) and taking into consideration (25), we have the generalized Klein-Gordon-Fock equation for vector particles (spin 1)

$$
\begin{gathered}
L=\left(D_{\mu} \chi\right)^{*}\left(D_{\mu} \chi\right)-\frac{1}{2}\left(\frac{m c}{\hbar}\right)^{2} \chi^{*} \chi-\frac{\lambda_{1}}{4}\left(\chi^{*} \chi\right)^{2}-\left(\frac{1}{2} \lambda_{2} G_{\mu v} G^{\mu \nu}\right)^{2} \\
D_{\mu}^{*}=\partial_{\mu}+i \Pi_{\mu} / \hbar, D_{\mu}=\partial_{\mu}-i \Pi_{\mu} / \hbar, \partial_{\mu} \Pi^{\mu}=0
\end{gathered}
$$

$\chi$ is the scalar potential, $\lambda_{1}$ and $\lambda_{2}$ are some ratios. This equation is invariant with respect to gauge transformations

$$
\chi \rightarrow \chi^{\prime}=\chi \mathrm{e}^{i \alpha} \text { and } \chi^{*} \rightarrow \chi^{\prime *}=\chi^{*} \mathrm{e}^{-i \alpha}
$$

has a specific solution

$$
\chi=(2 \pi)^{-3 / 2} \int \mathrm{d}^{4} k\left(K^{2}-\frac{m^{2} c^{2}}{\hbar^{2}}\right)\left(a(k) \mathrm{e}^{i k x}+a^{*}(k) \mathrm{e}^{-i k x}\right), k x=\boldsymbol{k} \boldsymbol{r}-\omega t
$$

These equations include two unknown potentials $\Pi, U$ which are defined by the solutions of Equations (28). We defined them very tentatively (1), which is not quite correct because it does not take into account the quantum structure of the particles. One possible way of generalizing it is expansion in Lie symmetry groups:

$$
\Pi \rightarrow \Pi_{\mu}^{\prime}=\Pi_{\mu}^{k} \tau_{k}, k=1,2,3
$$

where $\tau_{k}$ are the generators of these groups, they do not reverse, are Yang-Mills fields.

$$
\left|\tau_{i}, \tau_{k}\right|=e_{i k}^{n} \tau_{n}, i, k, n=1,2,3
$$

Each type of interaction is matched by a group consisting of $\tau_{k}=k^{2}-1$ matrices of order $k, U(1)$ corresponds to electromagnetic interaction, $S U(2)$ 
corresponds to the low-grade, $S U(3)$ corresponds to the high-grade etc.

The interaction medium is one particle $(k=1)$, a photon, no matrix is required to describe it $\left(\tau_{1}=0\right)$, the media of the low-grade one are three bosons $\left(\tau_{2}=3\right)$, three matrices of the second order are required to describe them $(k=2)$, like Pauli matrix, the media of the high-grade interaction are eight gluons $\left(\tau_{3}=8\right.$ ), eight matrices of the third order, like Gell-Mann matrices, and so on. Each type of interaction has its potential, for electromagnetic interaction the potential is long-range, for low-grade interaction it is short-range, for high-grade it is shifted etc. The interaction intensity can be assessed by the constant of their fine structure

$$
\alpha_{e m}=e^{2} / \hbar c=1 / 137, \alpha_{w}=g_{w}^{2} \approx 1 / 30, \alpha_{S}=g_{S}^{2} / \hbar c \approx 1 / 14
$$

The mass of the interaction medium is determined by Compton length $r_{C}=\hbar / m c$, for short-range forces $r_{C} \sim 10^{-16} \mathrm{~cm}$. According to this data, $m \sim 100$ giga electron volts, experimentally $m_{W}=80$ giga electron volts for charged bosons, and $m_{Z}=90$ giga electron volts for a neutral boson.

A similar, (analogiecliy) constant can also be introduced for gravitational interaction $\alpha_{g}=\gamma m_{g}^{2} / \hbar c$, however, unlike electrical $e$, weak $g_{w}$, and strong $g_{S}$ charges, the gravitational charge $m_{g}$, weighable and undefined, therefore it falls out of the field theory family and cannot be included in the Standard Model. The geometric representation of gravity further worsens the situation, quantizing becomes impossible. Plank's constant is a discrete value; the curvature is a continuous one; they are incompatible.

Thus, the kinetic field moment brings together all types of interaction and their reaction to the external forces, and it is easily quantized but in Equation (48) it is included in the second degree, and in case of second-degree representation part of information concerning the internal degrees of freedom, type of charge, spin, parity, and other quantum numbers are lost, and the coordinate conversion becomes meaningless. To preserve all the information, motion equations should be the first-degree equation system. Higher-degree equations should emerge as a result of a joint solution of the system.

\section{Conclusions}

Another mystery of creation, the mystery of space, has been unraveled. It has been established that space is neither vacuum nor a geometric shape but a force field able to resist external influences. By introducing the kinetic field moment, the Universe is transformed into a closed system, and everybody, as a member of this family, acquires an additional share of energy, kinetic moment, and angular momentum. Their sum is preserved

$$
m c^{2}+U=\text { const }, \quad \boldsymbol{p}+\Pi=\text { const }, \quad \boldsymbol{r} \times \boldsymbol{p}+\boldsymbol{r} \times \boldsymbol{\Pi}=\mathrm{const}
$$

Any change occurring in the IF space affects the condition of the body (environment), causing a responding reaction,

$$
\delta p_{\mu}=-\delta \Pi_{\mu}, \delta(\boldsymbol{r} \times \boldsymbol{p})=-\delta(\boldsymbol{r} \times \Pi)
$$


The Earth has preserved traces of numerous cataclysms which happened during its geological history: displacement of its magnetic and geographic poles, precession of the rotation axis, periodic climate change and much more. This influence has never ceased. It is continued now. For these cataclysms not to seem fatal, we should keep an eye on the IF dynamics, know its regular pattern, and find a way to apply this enormous force in practice.

\section{Funding}

The work has been supported by the Prince Aga Khan Development Foundation.

\section{References}

[1] Kittel, C.B., Knigt, W. and Ruderman, M. (2005) Mechanics. LAN, Moscow.

[2] Misner Charles, W., Thorne Kip, S. and Whiller John, A. (1973) Gravitation. Moscow.

[3] Chiu, H.-Y. and Hoffmann William, W. (1964) Gravitation and Relativity. Moscow.

[4] Heidi, F., et al. (2015) Journal of Modern Physics, 6, Article ID: 6069612.

[5] Sadykov, B. (2012) Journal of Modern Physics, 3, 155-162. https://doi.org/10.4236/jmp.2012.32021

[6] Ranku, K. (2015) Journal of Modern Physics, 6, 1007-1011. https://doi.org/10.4236/jmp.2015.67105

[7] Sadykov, B. (2017) Engineering Physics, 2, 3-12.

[8] Jacob, S. (2017) Journal of Modern Physics, 8, 181-250. https://doi.org/10.4236/jmp.2017.82016 